\title{
Video Article \\ Mapping the Emergent Spatial Organization of Mammalian Cells using Micropatterns and Quantitative Imaging
}

\author{
Darren Wisniewski ${ }^{1}$, Sally Lowell ${ }^{1}$, Guillaume Blin ${ }^{1}$ \\ ${ }^{1}$ MRC Centre for Regenerative Medicine, Institute for Stem Cell Research, School of Biological Sciences, University of Edinburgh
}

Correspondence to: Guillaume Blin at guillaume.blin@ed.ac.uk

URL: https://www.jove.com/video/59634

DOI: doi:10.3791/59634

Keywords: Developmental Biology, Issue 146, Patterning, micropattern, quantitative, stem cells, emergent behavior

Date Published: 4/30/2019

Citation: Wisniewski, D., Lowell, S., Blin, G. Mapping the Emergent Spatial Organization of Mammalian Cells using Micropatterns and Quantitative Imaging. J. Vis. Exp. (146), e59634, doi:10.3791/59634 (2019).

\section{Abstract}

A fundamental goal in biology is to understand how patterns emerge during development. Several groups have shown that patterning can be achieved in vitro when stem cells are spatially confined onto micropatterns, thus setting up experimental models which offer unique opportunities to identify, in vitro, the fundamental principles of biological organisation.

Here we describe our own implementation of the methodology. We adapted a photo-patterning technique to reduce the need for specialized equipment to make it easier to establish the method in a standard cell biology laboratory. We also developed a free, open-source and easy to install image analysis framework in order to precisely measure the preferential positioning of sub-populations of cells within colonies of standard shapes and sizes. This method makes it possible to reveal the existence of patterning events even in seemingly disorganized populations of cells. The technique provides quantitative insights and can be used to decouple influences of the environment (e.g., physical cues or endogenous signaling), on a given patterning process.

\section{Video Link}

The video component of this article can be found at https://www.jove.com/video/59634/

\section{Introduction}

In mammalian systems, patterning is an emergent property of the collective behavior of cells and so, patterns can form in vitro if appropriate cues are provided to the cells $s^{1,2,3,4,5,6}$. One way to reveal the intrinsic ability of the cells to self-organize in vitro is to force the cells to form groups/ colonies of a defined shapes and sizes ${ }^{7,8,9,10}$. A technique which enables this is micropatterning ${ }^{11}$. Micropatterning makes it possible to precisely define the location where extracellular matrix (ECM) molecules are deposited on a surface. This, in turn dictates where the cells can adhere and therefore controls how cells spatially organize.

Micropatterning is a technique with numerous applications, for example, micropatterning enables standardization of initial conditions prior to differentiation $^{12}$. Importantly, micropatterning makes it possible to easily control the size, shape and spacing of cell colonies and this property can be used to devise experiments aimed at interrogating the collective response of the cells to morphogen or to physical cues ${ }^{7,8,10,13,14,15,16,17}$.

Several micropatterning methods have been developed ${ }^{11}$. Photopatterning techniques are perhaps the easiest methods to establish ${ }^{18}$. These approaches also have the advantage of precision as they can be used to control the shape of single cells ${ }^{18,19,20}$. However, they also require expensive specialized equipment including a spin coater, a plasma chamber and a UVO (UV-Ozone) cleaner which are generally not readily available in standard biology laboratories. To facilitate adoption of the technique, we adapted the protocol to necessitate only the UVO lamp. We start from commercially available plastic slides which can be cut with scissors or with a hole punch to the desired format.

One important utility of micropatterns is the ability to standardize colonies in order to compare individual colonies across multiple replicates. This makes it possible to ask to what extent pattern formation within these colonies is reproducible, and to explore factors that influence the robustness of the patterning process. Importantly, quantification of "averaged" patterns across multiple standardized colonies can also reveal patterning processes that would not otherwise be apparent. The advantage of being able to quantify patterning on standardized colonies depends on being able to accurately measure protein expression, ideally at the single cell level. However, cells on micropatterns are often tightly packed, making them difficult to segment with high accuracy. Cells also often organize themselves in three rather than two dimensions, and it can be challenging to detect and preserve three-dimensional (3D) information during segmentation. Once cells have been successfully segmented, computational methods are needed for extracting patterning information from the resulting datasets.

We have developed segmentation and image analysis tools to help overcome these problems. This analysis method only uses free and opensource software and does not require knowledge of command line or programming to implement. To illustrate the method here, we use mouse embryonic stem (mES) cells which spontaneously express a marker of early differentiation brachyury (Tbra) ${ }^{21,22}$. While no apparent spatial arrangement is visually detectable, the method allows the creation of a map of the preferential positioning of T+ cells in colonies. We also show that Tbra patterning contrasts with the absence of a preferential localization of the cells expressing Id1, a direct readout of the bone 
morphogenetic protein (BMP) pathway ${ }^{23}$. We also discuss the current limitations of the method and how this technique may be adapted to other experimental systems.

Protocol

NOTE: An overview of the method is provided in Figure 1.

\section{Mask design}

1. Design the photomask according to the guidelines described in Azioune et al. ${ }^{18}$. See the Table of Materials for a reference to the software and mask manufacturer used for this study.

NOTE: Multiple geometries, sizes or spacing between shapes can be created on one mask. The UV lamp can fit a $15 \mathrm{~cm}$ photomask which may contain up to 49 different designs (assuming $2 \mathrm{~cm} \times 2 \mathrm{~cm}$ chips).

\section{Micropattern manufacturing procedure}

1. Prepare the necessary materials.

1. Prepare a solution of $0.1 \%$ poloxamer $407(10 \mathrm{mg}$ for $10 \mathrm{~mL})$ in phosphate buffered saline (PBS) and leave on a shaker at room temperature. The poloxamer 407 will take about 20 min to dissolve.

2. Lay laboratory film (see the Table of Materials) in the bottom of a $10 \mathrm{~cm}$ square Petri dish. This will be used as the chamber for matrix deposition.

3. Clean the surface of the photomask, first with $100 \%$ acetone, then with $100 \%$ isopropanol and finally with dd $\mathrm{H}_{2} \mathrm{O}$. If possible, air dry the photomask or otherwise dry the mask with clean paper towel.

4. Prepare a square, rigid and opaque piece of plastic with the exact same size as the photomask (later on referred as 'holder'). NOTE: This will be used to maintain plastic coverslips in contact with the photomask during the illumination step.

5. Turn the UVO lamp on and run a warming illumination for $10 \mathrm{~min}$

2. Create photopatterned chips.

NOTE: It is possible to adapt the procedure to create chips of any desired size. For simplicity, we describe here the procedure to generate a $12 \mathrm{~mm}$ round micropatterned chip.

1. Using a $12 \mathrm{~mm}$ hole punch, cut hydrophobic plastic slides to create $12 \mathrm{~mm}$ round coverslips and place them in a clean new Petri dish CAUTION: Use gloves at all time to avoid skin contact with the surface of the plastic as this may damage the surface treatment.

2. Carefully remove the protective film from the coverslips with tweezers. NOTE: Avoid damaging the plastic surface as this may influence the placement of the cells on the chip during the seeding procedure (section 3).

3. Place the photomask on a clean and stable surface (e.g., photomask box), chrome side facing up, and add a $2 \mu \mathrm{L}$ drop of ddH $\mathrm{H}_{2} \mathrm{O}$ at the position of the desired chip design.

4. Lay a coverslip onto the drop of $\mathrm{ddH}_{2} \mathrm{O}$ and press gently.

NOTE: Ensure that the plastic side which faces the photomask is the side which was protected by the film that was removed in the earlier step.

5. Place the holder on top of the plastic slides and carefully fix this sandwich with clamps in order to maintain the plastic pieces in contact with the photomask.

NOTE: Place the clamps as close as possible to the location of the plastic slides in order to ensure that the plastic slides are perfectly maintained in contact with the surface of the photomask.

6. Place the assembly in the UVO lamp at approximately $2 \mathrm{~cm}$ from the light source and illuminate for $10 \mathrm{~min}$. NOTE: The power of the light is estimated to be $6 \mathrm{~mW} / \mathrm{cm}^{2}$ at $254 \mathrm{~nm}$ of wavelength when the chip is placed at a distance of $2 \mathrm{~cm}$ from the source.

7. Hold the sandwich with the photomask at the bottom and carefully remove the clamps while maintaining pressure with one hand to prevent the slides from moving about while disassembling the sandwich. Remove the holder, ensuring that all plastic pieces are still on the mask and not stuck to the holder.

8. Add $\mathrm{ddH}_{2} \mathrm{O}$ on top of the chips and gently detach the chips from the photomask.

NOTE: If the plastic chip is stuck to the photomask, detach the chip using a plastic pipette tip to push the chip while holding tweezers slightly above the chip in case the chip detaches suddenly.

9. Finally, place the photopatterned chips within the matrix deposition chamber.

NOTE: Ensure that the illuminated side of the chip is facing upwards.

3. Deposit the matrix.

NOTE: All procedure in this section should be performed in a tissue culture hood.

1. Filter the poloxamer 407 solution through a $0.22 \mu \mathrm{m}$ polyethersulfone (PES) filter.

2. Prepare the ECM coating solution by mixing $500 \mu \mathrm{g} / \mathrm{mL}$ of sterile filtered poloxamer 407 and $1 \mathrm{mg} / \mathrm{mL}$ of gelatin.

NOTE: See also Table 1 for additional information regarding other possible ECM molecules.

3. Add $200 \mu \mathrm{L}$ of the coating solution onto each illuminated chip. The laboratory film will prevent the drop from falling outside of the chip.

4. Add a $3 \mathrm{~cm}$ Petri dish filled with $\mathrm{ddH}_{2} \mathrm{O}$ in order to limit evaporation and place it with the chip at $4{ }^{\circ} \mathrm{C}$ overnight. 


\section{Seeding procedure}

NOTE: The steps described below have been optimized for CGR8 mouse embryonic stem cells (mESC) ${ }^{24}$ using standard mESC medium (see also the Table of Materials). However, it is possible in principle to adapt the procedure for any cell type. Note also that conventional cell culture of mouse embryonic stem cells is not described here as extensive documentation can be found elsewhere ${ }^{25}$.

1. Aspirate the coating solution and incubate the chips twice for at least 5 min with sterile PBS.

2. In the meantime, prepare a cell suspension of $5.5 \times 10^{5} \mathrm{cells} / \mathrm{mL}$ in warm medium.

3. Pipet $200 \mu \mathrm{L}$ of cell suspension onto each chip $\left(\sim 100,000 \mathrm{cells} / \mathrm{cm}^{2}\right)$.

4. Close the seeding chamber and leave the cells to adhere for $1 \mathrm{~h}$ in the incubator.

5. After $1 \mathrm{~h}$, fill the wells of a multiwell plate (4-well or 24-well plate depending on the number of chips) with $500 \mu \mathrm{L} /$ well of warm medium and transfer the chips into the plate with sterile tweezers.

6. Shake the plate vigorously in order to detach non-adherent cells. Aspirate the medium and immediately replace with fresh warm medium. Check under the microscope to see if patterning is visible (Figure 2a).

NOTE: Adhesion time may need to be optimized when using cell lines other than mESC or other matrix proteins (see also Table 2).

7. Repeat this step until patterns become clearly visible as shown in Figure 2a. NOTE: This step is critical and determines the success of the procedure. A washing procedure which is too intense may detach the cells, in contrast insufficient washing may result in cells remaining attached in between the patterns (Figure 2c,d).

\section{Fixation}

NOTE: After $48 \mathrm{~h}$ in culture the cells should form dense colonies which rigorously follow the shape of the patterns (as shown in Figure $\mathbf{2 b}$ ).

1. Leaving the chips in the plate, remove $\sim 90 \%$ of the medium, leaving just enough medium to prevent the chips from drying NOTE: It is important that the chip never dries to avoid staining artefacts and to prevent cell detachment from the surface. Due to the hydrophobicity of the chip surface between adhesive patterns, the chip may have a tendency to de-wet. At this stage, the fixative may cause large domed colonies to detach from the chip. Washes should be very gentle, ideally performed by pipetting liquid on the side of the well and not directly onto the chip.

2. Add at least $500 \mu \mathrm{L}$ of paraformaldehyde (PFA)-based fixation solution per well and incubate for $10 \mathrm{~min}$. NOTE: If colonies appear particularly thick (more than 5 cell layers), it may be necessary to adjust fixation time to $20 \mathrm{~min}$.

3. After fixation, wash 3 times with the washing solution (PBS with $0.01 \%$ poloxamer 407 ). An extra wash using $50 \mathrm{mM} \mathrm{NH}_{4} \mathrm{Cl}$ diluted in washing solution may be intercalated to quench residual PFA cross-linking activity.

4. Incubate the samples for at least $30 \mathrm{~min}$ in blocking solution. NOTE: At this stage, samples may be stored at $4{ }^{\circ} \mathrm{C}$ for about a week prior to staining. If so, seal the plate with laboratory film to prevent evaporation.

\section{Immunostaining}

1. Prepare a staining chamber by placing a sheet of laboratory film in the bottom of a $10 \mathrm{~cm}$ square Petri dish.

2. Prepare antibody solutions (See Table $\mathbf{3}$ for a list of antibodies and dilutions used in this article).

3. Place the chip into the staining chamber with the side supporting the cells facing upwards and immediately add $100 \mu \mathrm{L}$ of primary antibody solution onto the chip.

CAUTION: At this stage, chips should not easily de-wet as in step 4.1. However, care should still be taken because it is important that the chips do not dry. If multiple chips have to be processed, apply step 5.3 to each chip sequentially.

4. Incubate for $1 \mathrm{~h}$ on a rotating platform at room temperature.

NOTE: If the cells have formed large 3D structures, a longer incubation time may be required to allow for uniform staining of the sample. Incubation time may be increased up to $24 \mathrm{~h}$. However, the staining chamber must contain a $3 \mathrm{~cm}$ dish filled with water and the staining chamber must be sealed with laboratory film to prevent evaporation.

5. Transfer the chips into a fresh multiwell plate and wash 3 times with the washing solution.

6. Perform incubation with secondary antibodies as described in step 5.3 and 5.4 .

7. Mount the chip on a microscopy slide using $20 \mu \mathrm{L}$ of any standard mounting medium (e.g., Mowiol).

\section{Imaging}

NOTE: Imaging can be performed on a standard confocal microscope. Here we only provide recommendations to ensure an image quality which will be sufficient for the subsequent quantitative analysis.

CAUTION: To avoid any operator bias, the colonies to image should only be chosen using the nuclear envelope signal (to see if a colony properly follows the shape of the pattern). Avoid checking the signal of markers of interest except when adjusting microscope settings.

1. Ensure that the acquisition bit depth is 12 or 16 bits.

2. Identify the appropriate settings to maximize the dynamic range for each image channel. In particular, avoid image clipping.

3. Include a channel to image the micropattern autofluorescence (see Figure 3).

4. Adjust Image size and zoom factor to obtain voxel sizes ranging between 0.1 and $0.6 \mu \mathrm{m}$ in the $\mathrm{x}$ - and $\mathrm{y}$-axes and ranging between 0.2 and 2 $\mu \mathrm{m}$ in the z-axis.

NOTE: For example, in this study, we used an inverted scanning confocal microscope with a 40x objective (numerical aperture equal to 1.3), an image size of $1024 \times 1024$ pixels without digital zoom and a z-step size of $0.5 \mu \mathrm{m}$. This resulted in a voxel size of $0.38 \mu \mathrm{m} \times 0.38 \mu \mathrm{m} \times 0.5$ $\mu \mathrm{m}$. 
5. For each colony, define the minimum and maximum position along the z-axis to ensure that the entire colony is acquired. At least one plane with low to no signal should be included below and above the colony.

6. Ensure that z-stack orientations are acquired consistently (either always top to bottom or always bottom to top)

7. Adjust the scanning speed, image resolution, frame averaging, and detector gains to identify an optimum between image quality and imaging time. As an indication, imaging time for one colony as shown in Figure 3 was approximately 2-3 min. Perform the image acquisition. CAUTION: All images, in order to be comparable, must be acquired on the same microscope with the same objective and acquisition settings.

8. At the end of the acquisition, save all the images and assign a unique naming convention to identify the experimental condition that each image represents.

NOTE: See Figure 4 as an example, this convention will be used later during the analysis procedure. Note that images may be saved to any format that is supported by BioFormats ${ }^{26}$. If colonies are larger than the field of view, the stitching plugin of Image J may be used ${ }^{27}$. Note also that in case of stitching, illumination roll off correction might be necessary.

\section{Image analysis}

NOTE: The recommended computer specifications for this procedure is: 16 GB RAM, a multi-core $3.33 \mathrm{GHz}$ CPU, and at least $50 \mathrm{~GB}$ of disk space (or more depending on the number of chips that have been imaged). The software has been tested on Linux, Windows and MacOS PickCells is a cross-platform image analysis application with a graphic user interface dedicated to the analysis of the collective organization of the cells in complex multidimensional images (Blin et al, in preparation). Note that more information on PickCells as well as documentation for the specific modules mentioned here can be found online: https://pickcellslab.frama.io/docs/. Note also that the interface is subject to change as we keep improving the software. If the interface differs from what is shown in the figure or video, please refer to the online manual.

1. Install and run PickCells following the documentation available online.

2. Import images and verify the accuracy of the provided information (Figure 4-1).

3. Document the name of each channel.

4. Segment nuclei based on the nuclear envelope signal using the Nessys module ${ }^{28}$ (Figure 4-2) and provide a prefix ("nuclei" for example) which will be used to name the generated segmented images.

NOTE: Documentations about usage and parameter adjustments can be found at https://framagit.org/pickcellslab/nessys.

5. Inspect and edit segmentations if necessary, using the segmentation editor module (Figure 4-3)

NOTE: If for any reason the segmentation process did not provide satisfactory results, manually delete images in the database folder and also delete the 'segmentation result' node in the MetaModel View. Then, repeat step 7.4 and 7.5. If the segmentation of only a small subset of images did not provide satisfactory results, then use the Nessys standalone application (see the link in 7.4), attempt segmentation on the 'faulty images' and replace the corresponding file in the database folder).

6. Segment the pattern autofluorescence signal using the basic segmentation module (Figure 4-4)

1. Provide a prefix ("pattern" for example) which will be used to name the generated segmented images.

2. Select the channel containing the autofluorescence signal.

3. Apply noise reduction; generally using a gaussian filter with a kernel size of $10 \times 10 \times 0.5$ voxels gives satisfactory results.

4. Set the lower threshold so that the background appears in blue while the foreground appears white. Set also the upper threshold to its maximum value to avoid high intensities being excluded from the final result (red areas).

5. Select skip for the last step.

6. Click finish and wait till all images are processed.

7. As for nuclei, segmentation results can now be visually inspected and corrected if necessary using the segmentation editor module (Figure 4-5).

8. Create nuclei objects and compute basic object features.

1. Launch the intrinsic features module from the task bar on the left of the main interface (Figure 4-6).

2. Close the Ellipsoid Fitter and Surface Extractor panels to keep only the Basic Features panel open.

3. Choose Nucleus as object type and choose the prefix given in step 7.4 for "segmented images".

4. Press Compute and wait till all the images have been processed.

NOTE: After this step, it will not be possible to edit the nuclei segmentations again.

9. Create pattern objects and compute basic object features. Repeat steps 7.8 .1 to 7.8 .4 , only this time choose Custom Type as object type and the prefix given in step 7.6.1 for segmented images.

NOTE: After this step, it will not be possible to edit the pattern segmentations again.

10. Store the name of the image each nucleus belongs to as a nucleus attribute.

1. Click on Data > New Attribute and select Nucleus in the popup dialog and click Ok

2. Select Collect data from other objects connected to the node and click Next.

3. In the left panel, select Image and then double click on the interrogation mark under the Finish flag in the Path definition panel to set the image node as target of the path.

4. Expand the Available Attributes pane on the left panel and select the name attribute.

5. Expand the Reduction operation pane and select Get One, then click on the change button and click Next.

6. Type "Image Name", press the tab key and click OK.

11. Create a "normalised coordinate" attribute in nuclei objects (Figure 4-7).

1. Adapt steps 7.10 .1 to 7.10 .6 to store the coordinates of the pattern centroid as a nucleus attribute. Name this new attribute "Pattern Coordinate".

2. Then, click on Data > New Attribute, select Nucleus and click Ok.

3. Select Define a function between spatial or directional vectors of the node and click Next.

4. For Type of function select Array Operation, for V1 select Item Vector and then Centroid, and for V2 select Item Vector and then Pattern Coordinate. 
5. Click Next, Type "Normalised Coordinate" in the Name field and click Finish.

12. Export the data to a tab separated value file.

\section{8. $R$ analysis}

1. Download and install Rstudio.

NOTE: Software information and download links are available at https://www.rstudio.com/.

2. Download the $\mathrm{R}$ scripts required for this analysis.

NOTE: Scripts can be downloaded from the GitLab repository: https://framagit.org/pickcellslab/hexmapr.

3. Open Rstudio.

NOTE: If running the scripts for the first time, install the required $\mathrm{R}$ packages (ggplot2 and scales).

4. From Rstudio, open the binnedmap_template.R script.

5. Set the working directory to the source file location.

6. Follow instructions provided in the script to adapt the script to any given dataset in order to obtain spatial maps as shown in Figure $\mathbf{5}$

7. Run the script to generate density maps.

\section{Representative Results}

The photo-patterning method described here makes it possible to precisely organize cultured cells into colonies of defined shapes and sizes. The success of this procedure should be clearly apparent immediately after the cell seeding procedure (step 3.7) as adhering cells will cluster according to the photomask design as shown in Figure $\mathbf{2 a}$. At $1 \mathrm{~h}$ after cell seeding, individual patterns may not be fully confluent (only a few cells per pattern), however, as the cells proliferate over time, patterns will become fully colonized with only very few cells outside the adhesive surfaces (Figure $\mathbf{2 b}$ ). The exact appearance of the culture will be cell line dependent. For example, mESC form domed-shape colonies ${ }^{10}$. A chip where patterning is not clear 1 to $2 \mathrm{~h}$ after cell seeding indicates failure of the procedure (Figure $\mathbf{2 c}, \mathbf{d}$ ).

Large and thick colonies can sometimes be challenging to stain homogeneously. We suggest to fix and permeabilize the cells in one step (section 4) as this can improve antibody penetration ${ }^{29}$. This is the reason why the chosen fixative solution contains a detergent. Figure 3 shows the fluorescence signal which is expected after immunostaining. Notice that bright Id1 positive cells are found within dense regions (bright NPC regions) of the colonies (Figure $\mathbf{3 A}$ ). Hints such as this are useful to assess the quality of the antibody staining procedure. Notice also that the micropatterns created with the present technique are autofluorescent. This signal (Figure 3A,B left most images) is useful during the analysis stage to spatially register colonies with one another and create the results shown in Figure $\mathbf{5}$. The autofluorescence signal is generally the brightest when the sample is excited with a $405 \mathrm{~nm}$ laser and this channel should be left without staining for this purpose. Figure 3 also shows how the cells are precisely constrained on patterns of different shapes.

The analysis of imaging data is performed in PickCells, a free and open-source software developed in our lab (Blin et al., in preparation). This software includes the image analysis modules to read and sort confocal images (Figure 4-1), to segment (Figure 4-2,4-4) and curate segmented objects (Figure 4-3,4-5), to compute object features such as coordinates or average intensity (Figure 4-6) and to export the data (Figure 4-7,4-8). Importantly, we developed a robust nuclear segmentation method called Nessys ${ }^{28}$ which is particularly suited for dense and heterogeneous populations of cells such as cells grown on micropatterns (Figure 3). Figure 4-2 shows a representative output of the Nessys module where each individual cell is accurately given a unique color identity. Only minimal editing should be necessary, however editing is feasible should the user decide so (Figure 4-3). Finally PickCells provides a number of visualization modules to visualize the data. An example is given in Figure 4-6: a ring-shaped colony is rendered in 3D where nuclei are color-coded according to their position along the z-axis. Once the analysis is validated in PickCells, data can be exported to create the spatial maps in R using the scripts available at (https://framagit.org/ pickcellslab/hexmapr) as shown in Figure $5^{30}$.

We have shown recently that spatial confinement of $\mathrm{mESC}$ on small $\left(30,000 \mu \mathrm{m}^{2}\right)$ disc or ellipse micropatterns guides the patterning of a subpopulation of cells expressing the mesodermal marker Tbra ${ }^{10}$. Thus, to illustrate our method here, we ask if patterning of Tbra may be influenced by BMP signaling in larger colonies $\left(90,000 \mu \mathrm{m}^{2}\right)$. Figure 5A shows that when mESC are grown on large disc micropatterns, Tbra+ cells are preferentially restricted to the periphery of the pattern (Tbra+ density map), where the local cell density is the lowest (see the blue map on the left of Figure 5A). This patterning of Tbra is confirmed by the map of mean Tbra intensity.

These data demonstrate that the method can reveal sub-visual information. Indeed, from Figure 3, visual inspection of one colony is not sufficient to identify any form of spatial organization in Tbra expression. This is notably explained by the important colony to colony variability which is quantified and shown in the right-most panel of Figure 5A.

The technique also shows that no detectable patterning exists for Id1 (a target of BMP signaling) which may indicate that T patterning is not driven by BMP signaling in this context.

Micropatterning makes it possible to force colonies to adopt almost any desired geometry. This is particularly useful to interrogate how the system responds to various geometries. For example, we may reason that if a morphogen gradient builds up at the center of the colony, creating a hole in the colony would disrupt this gradient. Interestingly we still observe patterning on a ring micropattern albeit in a less robust manner (Figure 5B). 


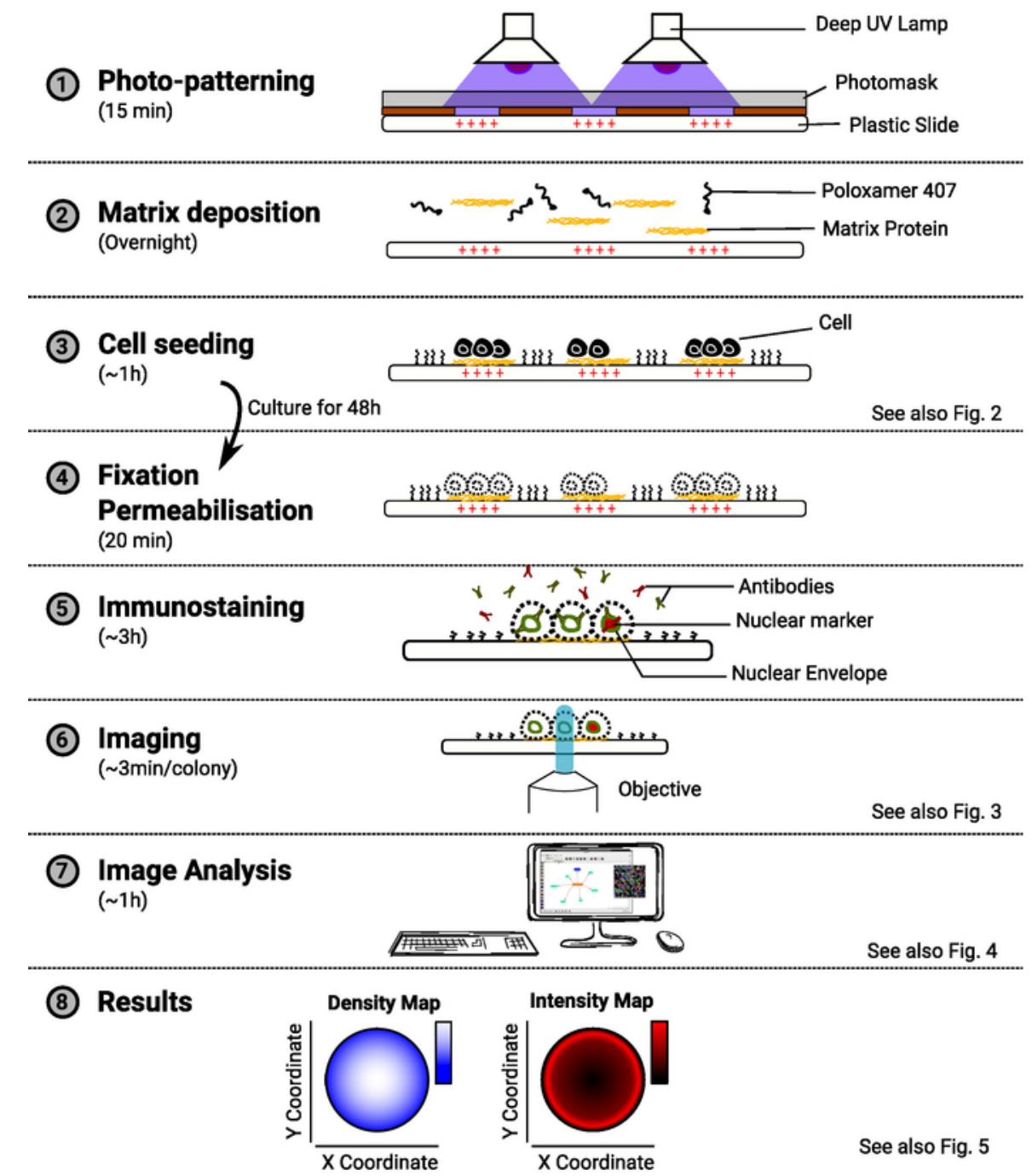

Figure 1: Overview of the method. The diagram showing the main steps of the method. For each step, the estimated amount of time is indicated under the name of the task and a schematic illustrates the purpose of the procedure. A reference to a relevant figure is also provided when available. Please click here to view a larger version of this figure. 

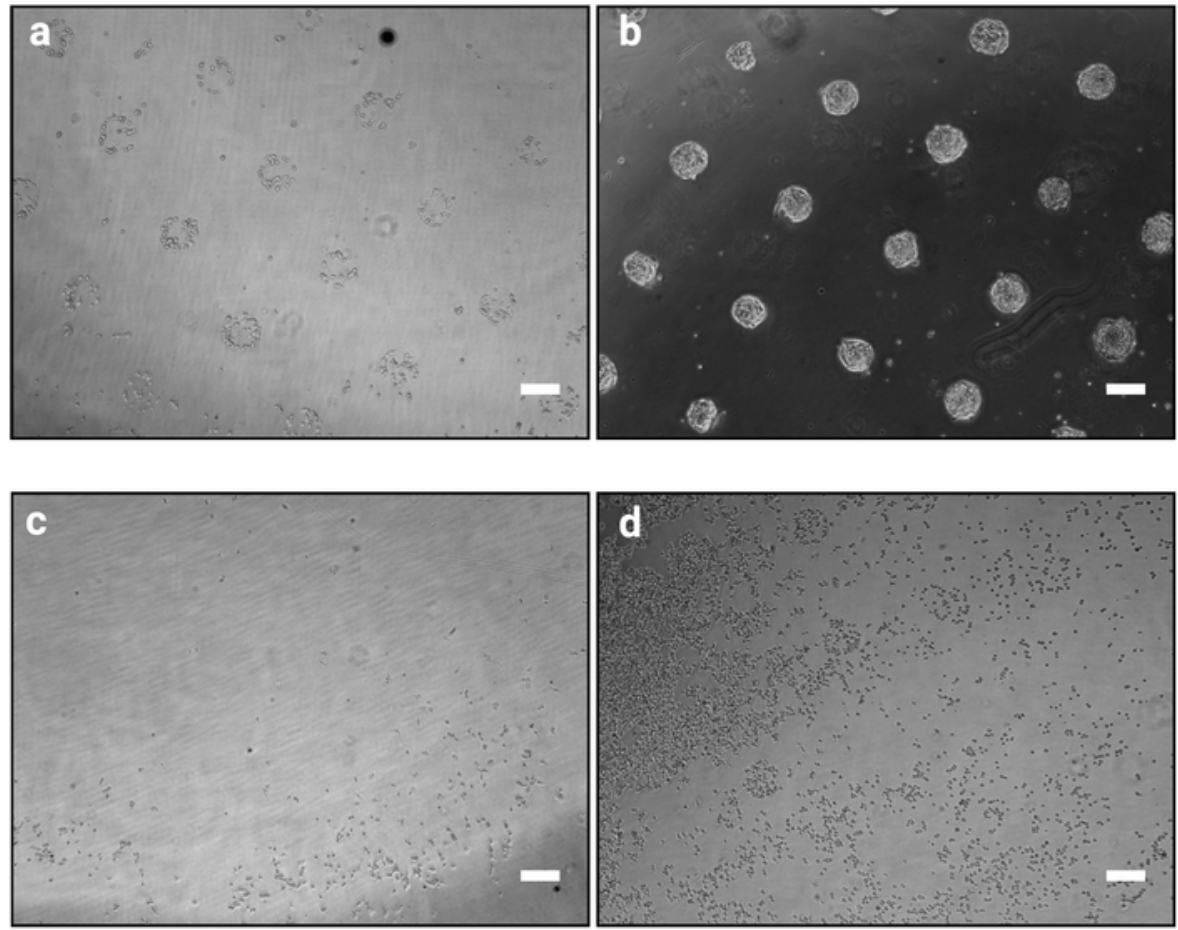

Figure 2: Culture appearance $1 \mathrm{~h}$ and $48 \mathrm{~h}$ after seeding the cells on micropatterns. Brightfield images of mESC seeded onto micropatterns. (a) Expected cell organization $1 \mathrm{~h}$ after seeding, patterns should be clearly identifiable. (b) Expected result after $48 \mathrm{~h}$ of cultures. mESC have proliferated and are still strictly confined to the pattern shapes. (c-d) Possible non-optimal outcomes, either very few cells adhere to the plastic except at the periphery of the slide (c) or cells adhere in between the patterns (d). See Table 2 for a troubleshooting guide. Please click here to view a larger version of this figure. 

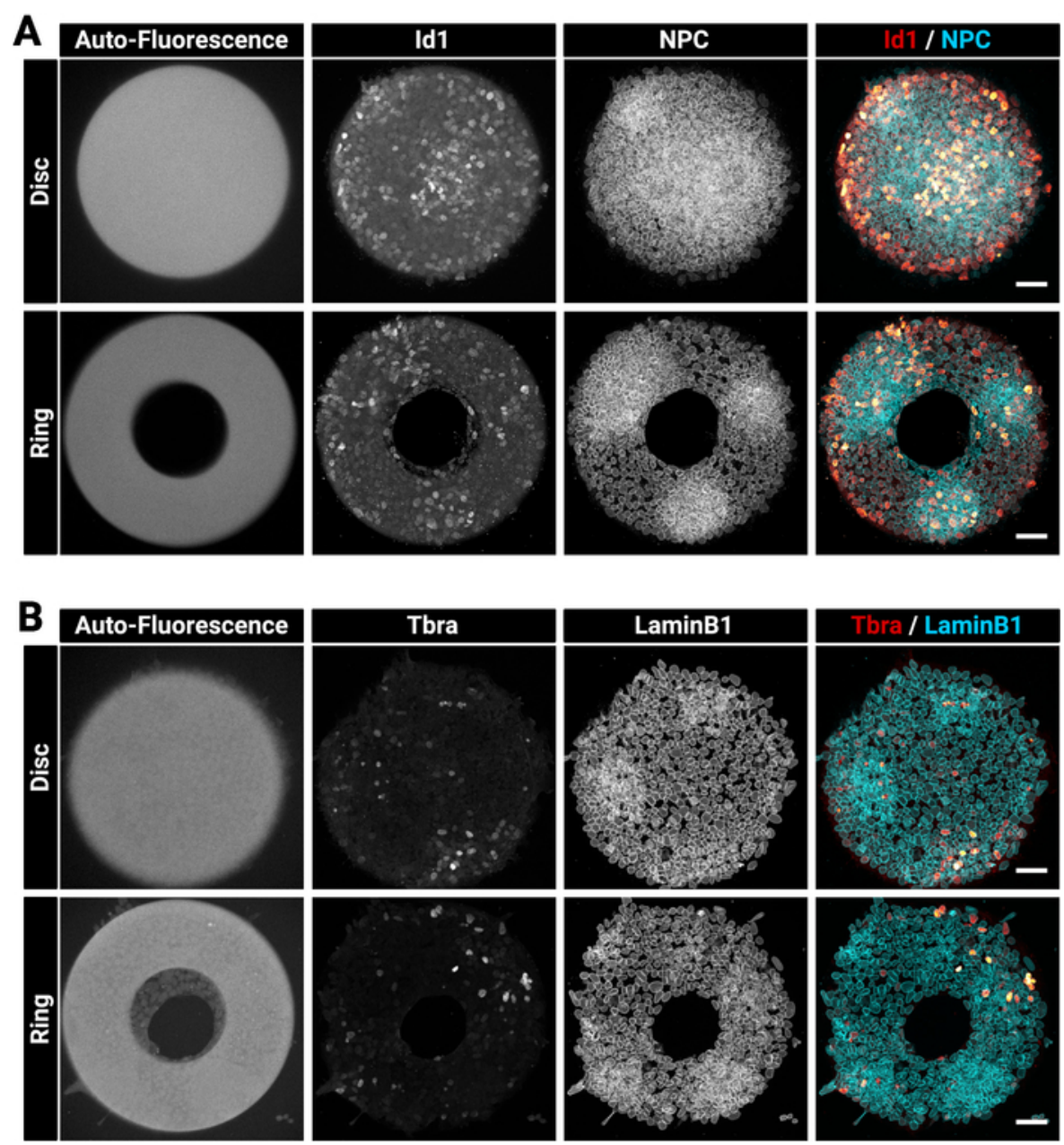

Figure 3: Representative confocal images of immunostained colonies grown on micropatterns.

Representative mESC colonies after immunofluorescence for (A) Id1 and Nuclear Pore Complex or (B) Tbra and LaminB1. For each staining, a colony grown on a disc micropattern and a colony grown on a ring micropattern is shown. Individual channels are provided as gray scale images. Notice the clear auto-fluorescence signal of the micropattern ( $405 \mathrm{~nm}$ laser excitation). Scale bar represents $50 \mu \mathrm{m}$. Please click here to view a larger version of this figure. 


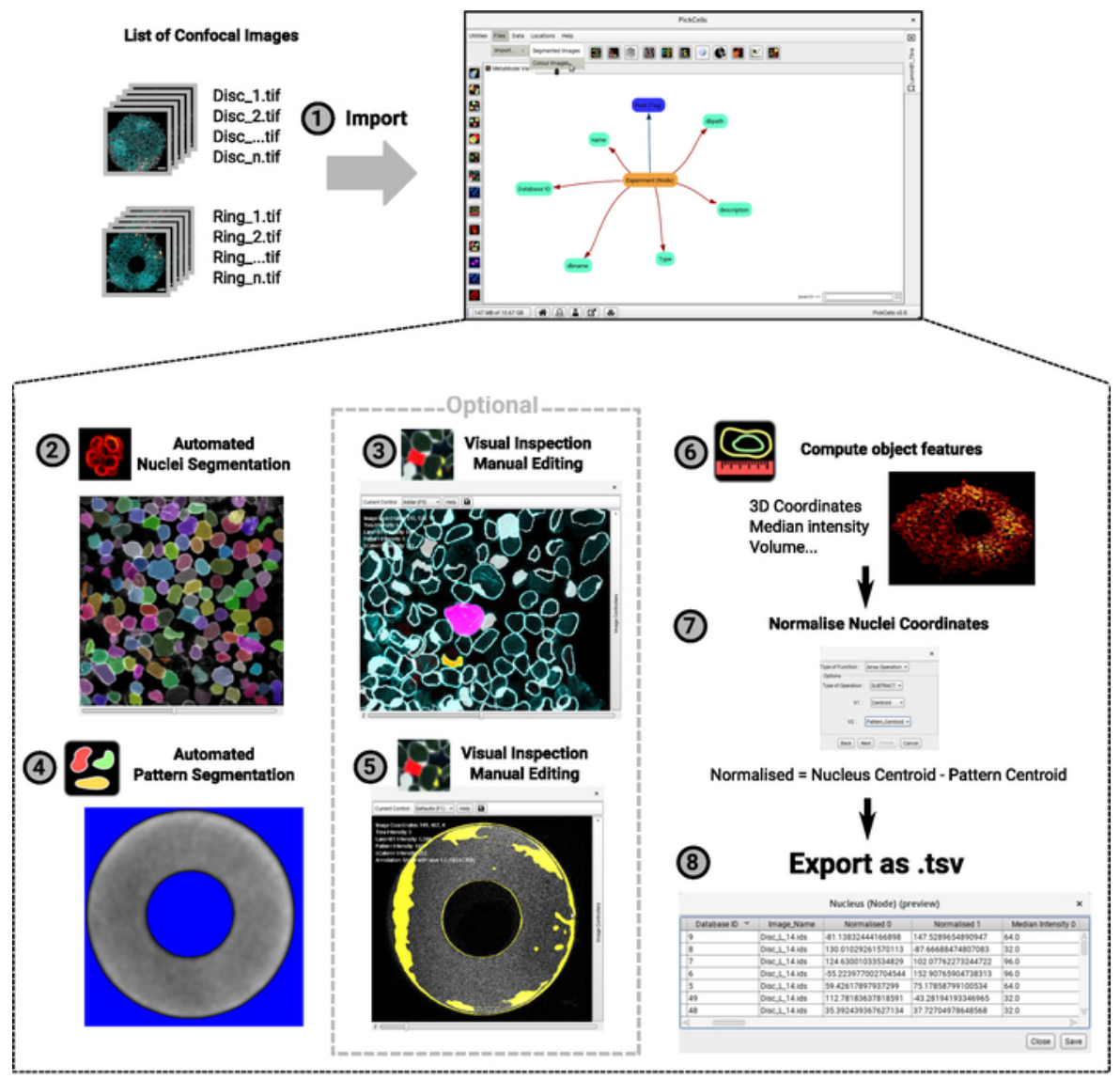

Figure 4: Flow chart of the image analysis procedure. A list of 3D confocal images is imported into PickCells for analysis (1). This example shows an experiment with two distinct shapes (discs and rings as in Figure 2). The image naming convention is shown on the left and PickCells interface on the right. Then, the Nessys module is used to automatically segment nuclei (2). In the screenshot, each individual nucleus is given a unique color indicating accurate segmentation. The autofluorescence of the pattern is also segmented, this time, using the 'basic segmentation' module (4). Background appears in blue and white signal will be defined as the pattern shape. Segmented shapes are then visually inspected to ensure accurate segmentation, and edited if required using the segmentation editor module (3-5). The screenshots show the outline of the detected shapes. The pink and yellow shapes have been edited. Finally, objects features are computed and exported to file to be later processed into $R(6-7)$. A screenshot of a colony rendered as a 3D view is provided (6). For steps 2 to 6 the icons found in the PickCells interface at the time of writing this article are given next to the step index. Please click here to view a larger version of this figure. 
A

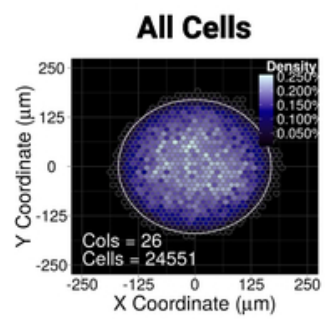

B

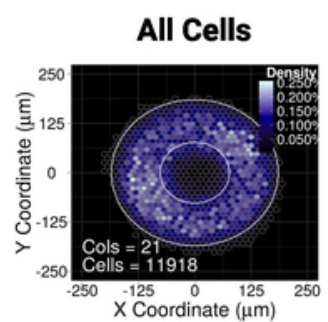

Density
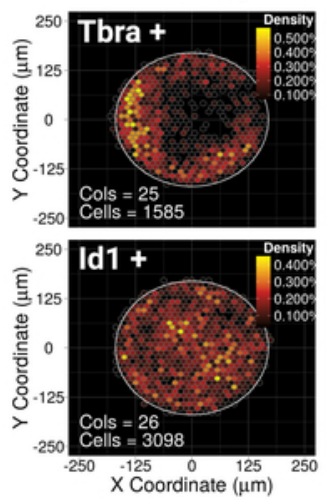

Density
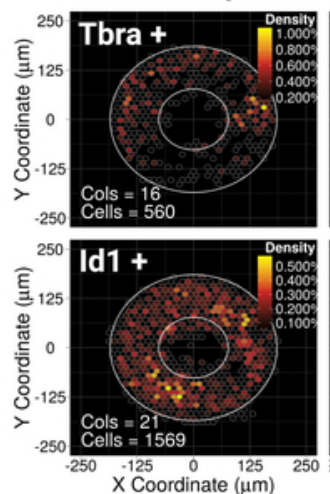

Mean Intensity
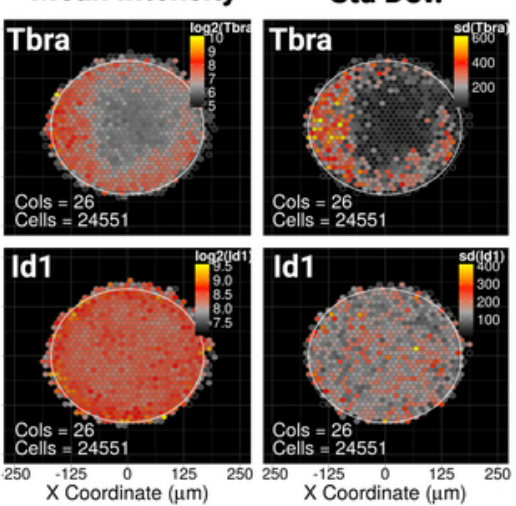

Mean Intensity

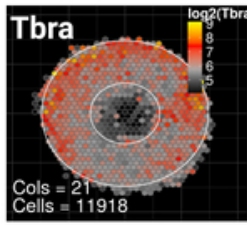

\section{Std Dev.}
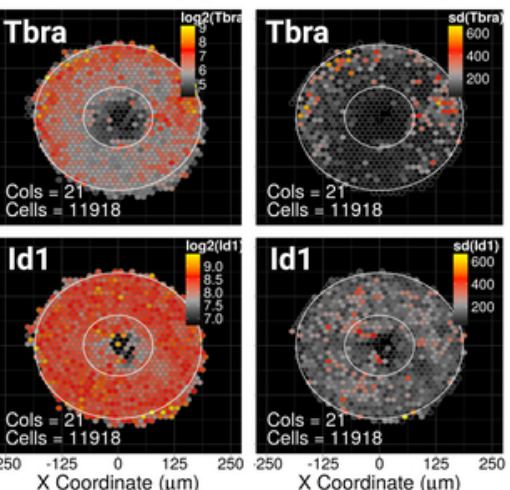

Figure 5: Representative results for two different transcription factors and micropattern shapes

Binned spatial map for mESC grown for $48 \mathrm{~h}$ on (A) disc shaped micropatterns or (B) ring shaped micropatterns. For each micropattern shape, the map of cell density, irrespective of cell phenotype, is shown on the left with a blue color scale. Then for each marker (Tbra on the top row and Id 1 on the bottom row), three distinct maps are provided, from left to right: cell density map of marker expressing cells only (threshold based analysis), the map of the average marker intensity (log2) and the map of the standard deviation of the marker intensity. Intensities are given as arbitrary fluorescence units. For each map, the micropattern shape is given as a white outline. Please click here to view a larger version of this figure.

\begin{tabular}{|l|l|l|l|l|}
\hline & ECM type & Gelatin & Fibronectin & $\begin{array}{l}\text { Basement Membrane } \\
\text { Matrix }\end{array}$ \\
\hline \multirow{5}{*}{ Concentration } & ECM concentration & $1 \mathrm{mg} / \mathrm{mL}$ & $20 \mu \mathrm{g} / \mathrm{mL}$ & $200 \mu \mathrm{g} / \mathrm{mL}$ \\
\cline { 2 - 5 } & $\begin{array}{l}\text { Poloxamer } 407 \\
\text { concentration }\end{array}$ & $500 \mu \mathrm{g} / \mathrm{mL}$ & $400 \mu \mathrm{g} / \mathrm{mL}$ & $1 \mathrm{mg} / \mathrm{mL}$ \\
\hline \multirow{3}{*}{ Tested with } & mESC & YES & YES & YES \\
\cline { 2 - 5 } & mEpiSC & NO & YES & YES \\
\cline { 2 - 5 } & Serum free medium & NO & YES & YES \\
\hline
\end{tabular}

Table 1: Tested concentrations of poloxamer 407 and ECM. This table provides an overview of the concentrations of ECM and poloxamer 407 that we tested in our lab. For each ECM/poloxamer 407 combination, the cell type for which patterning was successfully achieved is shown as well as whether the culture contained serum or not. $\mathrm{mESC}=$ mouse embryonic stem cell, $\mathrm{mEpiSC}=$ mouse epiblast stem cell. 


\begin{tabular}{|c|c|c|c|}
\hline Procedure & Observation & Possible Issue & Solution \\
\hline \multirow[t]{8}{*}{ Micropatterning } & \multirow[t]{3}{*}{ Low cell attachment } & $\begin{array}{l}\text { inappropriate ECM/poloxamer } 307 \\
\text { concentration ratio }\end{array}$ & $\begin{array}{l}\text { Increase the ECM/poloxamer } 307 \\
\text { concentration ratio }\end{array}$ \\
\hline & & Cell attachment time too short & $\begin{array}{l}\text { Increase incubation time to give } \\
\text { enough time to the cells to properly } \\
\text { adhere to patterns (step 3.4). To } \\
\text { optimise this step, checking the } \\
\text { cells under a microscope can help } \\
\text { detect a change in cell morphology } \\
\text { indicating that the cells have } \\
\text { started to adhere. }\end{array}$ \\
\hline & & Washes too intense (step 3.6) & $\begin{array}{l}\text { When replacing medium, avoid } \\
\text { pipetting medium directly onto the } \\
\text { chips. Instead, gently pipet the } \\
\text { medium on the walls of the well } \\
\text { instead }\end{array}$ \\
\hline & \multirow[t]{3}{*}{ Cells adhere between the patterns } & $\begin{array}{l}\text { inappropriate ECM/poloxamer } 307 \\
\text { concentration ratio }\end{array}$ & $\begin{array}{l}\text { Decrease the ECM/poloxamer } 407 \\
\text { concentration ratio }\end{array}$ \\
\hline & & Cell attachment time too long & $\begin{array}{l}\text { Decrease incubation time (step } \\
3.4 \text { ). }\end{array}$ \\
\hline & & Washes ineffective (step 3.6) & $\begin{array}{l}\text { Shaking the plate vigorously is } \\
\text { usually sufficient to detach the cells } \\
\text { in excess. For cell types which } \\
\text { tend to adhere strongly to the chip, } \\
\text { pipetting directly onto the chip may } \\
\text { improve the outcome. Increasing } \\
\text { the number of washes may also } \\
\text { help, notably to ensure that no cell } \\
\text { remain floating in the medium after } \\
\text { this step. }\end{array}$ \\
\hline & \multirow[t]{2}{*}{$\begin{array}{l}\text { Cells do not strictly follow the } \\
\text { pattern shape }\end{array}$} & $\begin{array}{l}\text { 'Incompatible' cell type and pattern } \\
\text { geometry }\end{array}$ & $\begin{array}{l}\text { Plan/design multiple geometries/ } \\
\text { sizes to be added onto the } \\
\text { photomask to be able to test and } \\
\text { identify the optimal pattern size } \\
\text { for a given pattern shape and cell } \\
\text { type. Please, see the 'Limitations' } \\
\text { section in the discussion }\end{array}$ \\
\hline & & $\begin{array}{l}\text { Non-optimal photo-patterning, this } \\
\text { can be diagnosed by observing the } \\
\text { sharpness of the autofluorescence } \\
\text { signal. Pattern boundaries should } \\
\text { look sharp as in Fig. } 2 \text {. If the } \\
\text { borders of the patterns appear } \\
\text { blurred then the photo-patterning } \\
\text { step needs to be improved. }\end{array}$ & $\begin{array}{l}\text { Blurred pattern edges indicate } \\
\text { that the plastic slide was not close } \\
\text { enough to the surface of the mask } \\
\text { during the illumination step. Ensure } \\
\text { that the pieces holding the slides to } \\
\text { the photomask are even and that a } \\
\text { constant and sufficient pressure is } \\
\text { applied to the assembly during the } \\
\text { illumination procedure. }\end{array}$ \\
\hline \multirow[t]{4}{*}{ Staining } & \multirow[t]{2}{*}{ Non-homogeneous staining } & antibody incubation time too short & $\begin{array}{l}\text { Increase antibody incubation time } \\
\text { (up to } 24 \mathrm{~h} \text { at room temperature) }\end{array}$ \\
\hline & & $\begin{array}{l}\text { colonies flattened during the } \\
\text { mounting procedure }\end{array}$ & $\begin{array}{l}\text { Mount micropattern slides in a } \\
\text { chamber such as chamlide or } \\
\text { cytoo-chambers to perform both } \\
\text { immunostaining and imaging } \\
\text { without the need for mounting the } \\
\text { cells. This will better preserve the } \\
\text { colonies 3D-structure. }\end{array}$ \\
\hline & $\begin{array}{l}\text { Detaching colonies during the } \\
\text { staining procedure }\end{array}$ & Dewetting of the chip & $\begin{array}{l}\text { Leave enough medium or use } \\
2 \text { pipettes, one to remove the } \\
\text { medium and the other to add the } \\
\text { fresh solution }\end{array}$ \\
\hline & $\begin{array}{l}\text { Colonies appear sheared under the } \\
\text { microscope }\end{array}$ & $\begin{array}{l}\text { colonies were sheared while } \\
\text { mounting the chip on the } \\
\text { microscopy slide }\end{array}$ & $\begin{array}{l}\text { Be very gentle when mounting } \\
\text { the chips. Alternatively, mount } \\
\text { micropatterns slides in a } \\
\text { chamber such as chamlide or }\end{array}$ \\
\hline
\end{tabular}


cytoochambers to perform both immunostaining and imaging without the need for mounting the cells. This also preserves the colony ultrastructure.

Table 2: Troubleshooting guide. This table provides an overview of possible sub-optimal outcomes. The potential sources of the problems are also listed together with recommended solutions.

\section{Discussion}

Here we describe a method for analyzing emergent patterning in cultures of cells. A simplified micropatterning approach is used for standardizing the shape and size of cell colonies, and we present image analysis tools and R scripts that enable the detection and quantification of patterns within these colonies.

The pipeline we propose is similar to some extent with a previously published method ${ }^{31}$ where the authors focus on the culture conditions, using commercially available micropatterns, to obtain reproducible germ layer formation in ESC colonies for the study of early gastrulation events in vitro. Our aim is more focused towards providing a generalizable pipeline for the discovery of pattern formation in vitro where the collective organization of the cells may only become apparent after statistical analysis. For this reason, we provide a robust image analysis workflow which enables the accurate identification and analysis of nuclear position in 3D space over multiple colonies (see also the 'Advantage and limitations of the pattern detection method' section of this discussion). We also decided to develop a simple in-house micropatterning approach which offers a more flexible and cheaper alternative to commercially available solutions in the long term which we hope will be useful to the community.

Finally, we note that during the revision of this manuscript, a new package for the analysis of patterning in vitro similar to our R scripts has been released $^{32}$. This new package accepts tables of cell features as input which can be obtained from high throughput imaging platforms. We believe that the table of nuclei features generated at step 7 of our protocol could in principle serve as an input to this new package although we have not tested this possibility ourselves.

\section{Adaptability of the method to other cell types and colony geometries}

We present this approach in the context of studying emergence of mesodermal transcription factors in cultures of pluripotent cells in the presence of serum. However, the method is readily adaptable to other cell types and to serum-free cultures although it may be necessary to optimize ECM/poloxamer 407 concentrations (see Table 1 for tested concentrations and Table 2 for a troubleshooting guide). The method can also be adapted to larger or smaller sizes of micropatterns and to a wide range of shapes according to the needs of the user. However, while establishing the method, it is important to be aware that not all shape/cell type combinations are optimal. For example, mESC express high levels of E-cadherin ${ }^{33,34}$ enabling these cells to form collective structures spanning areas that are devoid of ECM. These cells do not strictly follow geometries with sharp angles or that include small holes in the pattern. Notice for example that on the ring of Figure 3B, the cells are in the process of colonizing the central area. In our hands a smaller central area did not force mESC to form ring-shaped colonies. It is therefore highly recommended to include a diversity of geometries while designing the photomask to be able to test and identify the optimal sizes and curvatures which will be suited for the cell type of choice.

Another important factor to take into consideration is the length of the experiment and the proliferation rate of the cells. For some rapidly proliferating cell types (including pluripotent cells) it can be difficult to maintain cells on micropatterns over many days (For mESC three days is a maximum). Also, seeding of cells on micropatterns does not always occur optimally for every colony, so it is advisable to seed an excess of colonies in order to have spares.

\section{Advantage and limitations of the pattern detection method}

One particular advantage of the method is the ability to detect "averaged" patterns by combining image analysis results from multiple replicate colonies (Figure 5). This can reveal patterning events that are not apparent from inspection of individual colonies. A disadvantage of this 'averaging' approach is that it may miss certain types of repetitive patterns, for example small spots or narrow stripes. However, these types of pattern may instead be revealed with a combination of carefully chosen pattern sizes ${ }^{8}$. Also, the image analysis pipeline described here provides quantitative data at both single cell and colony resolution offering the possibility to investigate the level of inter-colony variability (Figure 5) or to perform neighbor analysis at multiple scales ${ }^{10}$.

Another important advantage of the averaging method is that it offers the opportunity to map the preferential location of many markers without being limited by available fluorophores of detection channels. Indeed, although we make use of only two markers of differentiation in the work presented here, the ability to standardize colonies and extract "averaged" patterns makes it possible to compare the distribution maps from different sets of colonies together in order to reveal the generalized spatial relationships of markers to each other.

Furthermore, although our focus here has been to study markers of differentiation, the analysis method can be extended to study other biological processes for which nuclear markers are available. For example micropatterning of a cell line containing a fluorescence ubiquitination cell cycle indicator $^{35}(\mathrm{FUCCl})$ would make it possible to study how colony level geometry may influence cell cycle events in the group.

\section{Future directions}

The method is amenable to medium throughput image analysis, however, image acquisition is currently not fully automated and can become limiting for very large experiments. The regular arrangements of colonies should make it possible to create fully automatized acquisition routines similar to what has been developed for single cell averaging ${ }^{20}$. However, because the size of the field required to image a colony is large, possibly requiring mosaicking, and because colonies are three-dimensional, it is highly desirable to reduce both dataset size and acquisition 
time by imaging only relevant colonies. Therefore, future efforts may be dedicated to develop an 'intelligent' microscope able to identify relevant colonies and adapt imaging coordinates to each sample. This will not only reduce time and effort but also prevent potential operator biases.

The analysis pipelines may also be made more efficient by reducing the number of steps the user needs to take. We have plans to build a pipeline-building mechanism and to integrate $\mathrm{R}$ directly into our software (see also issues pickcells-api\#3 and pickcells-rjava\#1 in the issue tracker of our code repositories [https://framagit.org/groups/pickcellslab/-/issues]). The full automation of the analysis procedure will reduce time and effort and limit potential user errors.

Finally, we note that our analysis method does not yet fully capture the dynamic nature of cellular patterning. Some limited dynamic information can be extracted by examining a time-series of snapshot images ${ }^{8,10,36}$. However, being able to record the history of the cell population is highly desirable if we wish to better understand how patterning emerges. One limitation is that accurate tracking of individual cells in a 3D dense cell population remains a very challenging task ${ }^{37}$. Our cell detection method uses the nuclear envelope and performs particularly well on dense and overlapping cell populations ${ }^{28}$. Live reporters of the nuclear envelope are readily available ${ }^{28,38}$ and one advantage of the micropatterning technique is that it may be used to prevent the cells from moving outside the field of view during long-term imaging. Overall, we are confident that automated tracking of cells will be achievable using a combination of recently established tools ${ }^{28,39,40}$ and that this should bring new insights into the fundamental principles of self-organization.

\section{Disclosures}

The authors have nothing to disclose

\section{Acknowledgments}

This work was funded by a Sir Henry Wellcome post-doctoral fellowship (WT100133 to G.B.), a Wellcome Trust Senior Fellowship (WT103789AIA to S.L.), and a Wellcome Trust PhD studentship to (108906/Z/15/Z to D.W.). We are also grateful to Dr Manuel Thery for his advice on adapting the photopatterning technique.

\section{References}

1. Sasai, Y. Cytosystems dynamics in self-organization of tissue architecture. Nature. 493 (7432), 318-326 (2013).

2. Turner, D.A., Baillie-Johnson, P., Martinez Arias, A. Organoids and the genetically encoded self-assembly of embryonic stem cells. BioEssays. 38 (2), 181-191 (2016).

3. Simunovic, M., Brivanlou, A.H. Embryoids, organoids and gastruloids: new approaches to understanding embryogenesis. Development. 144 (6), 976-985 (2017).

4. Tewary, M., Shakiba, N., Zandstra, P.W. Stem cell bioengineering: building from stem cell biology. Nature Reviews. Genetics. 19 (10), 595-614 (2018).

5. Shahbazi, M.N., Zernicka-Goetz, M. Deconstructing and reconstructing the mouse and human early embryo. Nature Cell Biology. 20 (8), 878 (2018).

6. Laurent, J. et al. Convergence of microengineering and cellular self-organization towards functional tissue manufacturing. Nature Biomedical Engineering. 1 (12), 939 (2017).

7. McBeath, R., Pirone, D.M., Nelson, C.M., Bhadriraju, K., Chen, C.S. Cell shape, cytoskeletal tension, and RhoA regulate stem cell lineage commitment. Developmental Cell. 6 (4), 483-495 (2004).

8. Tewary, M. et al. A stepwise model of Reaction-Diffusion and Positional-Information governs self-organized human peri-gastrulation-like patterning. Development. (2017).

9. Warmflash, A., Sorre, B., Etoc, F., Siggia, E.D., Brivanlou, A.H. A method to recapitulate early embryonic spatial patterning in human embryonic stem cells. Nature Methods. 11 (8), 847-854 (2014).

10. Blin, G., Wisniewski, D., Picart, C., Thery, M., Puceat, M., Lowell, S. Geometrical confinement controls the asymmetric patterning of brachyury in cultures of pluripotent cells. Development. 145 (18), dev166025 (2018).

11. Micropatterning in cell biology. Pt. A. Elsevier. Amsterdam. (2014).

12. Bauwens, C.L. et al. Control of human embryonic stem cell colony and aggregate size heterogeneity influences differentiation trajectories. Stem Cells (Dayton, Ohio). 26 (9), 2300-2310 (2008).

13. Heemskerk, I., Burt, K., Miller, M., Chabra, S., Guerra, M.C., Warmflash, A. Morphogen dynamics control patterning in a stem cell model of the human embryo. bioRxiv. 202366 (2017).

14. Nemashkalo, A., Ruzo, A., Heemskerk, I., Warmflash, A. Morphogen and community effects determine cell fates in response to BMP4 signaling in human embryonic stem cells. Development. 144 (17), 3042-3053 (2017).

15. Peerani, R., Onishi, K., Mahdavi, A., Kumacheva, E., Zandstra, P.W. Manipulation of signaling thresholds in "engineered stem cell niches" identifies design criteria for pluripotent stem cell screens. PloS One. 4 (7), e6438 (2009).

16. Peerani, R. et al. Niche-mediated control of human embryonic stem cell self-renewal and differentiation. The EMBO journal. 26 (22), 4744-4755 (2007).

17. Etoc, F. et al. A Balance between Secreted Inhibitors and Edge Sensing Controls Gastruloid Self-Organization. Developmental Cell. 39 (3), 302-315 (2016).

18. Azioune, A., Carpi, N., Tseng, Q., Théry, M., Piel, M. Protein micropatterns: A direct printing protocol using deep UVs. Methods in Cell Biology. 97, 133-146 (2010).

19. Théry, M. Micropatterning as a tool to decipher cell morphogenesis and functions. Journal of Cell Science. 123 (Pt 24), $4201-4213$ (2010).

20. Degot, S. et al. Improved Visualization and Quantitative Analysis of Drug Effects Using Micropatterned Cells. JoVE (Journal of Visualized Experiments). (46), e2514 (2010). 
21. Beddington, R.S.P., Rashbass, P., Wilson, V. Brachyury - a gene affecting mouse gastrulation and early organogenesis. Development. 116 (Supplement), 157-165 (1992).

22. Wilkinson, D.G., Bhatt, S., Herrmann, B.G. Expression pattern of the mouse T gene and its role in mesoderm formation. Nature. 343 (6259), 657-659 (1990).

23. Hollnagel, A., Oehlmann, V., Heymer, J., Rüther, U., Nordheim, A. Id Genes Are Direct Targets of Bone Morphogenetic Protein Induction in Embryonic Stem Cells. Journal of Biological Chemistry. 274 (28), 19838-19845 (1999).

24. Mountford, P. et al. Dicistronic targeting constructs: reporters and modifiers of mammalian gene expression. Proceedings of the National Academy of Sciences of the United States of America. 91 (10), 4303-4307 (1994).

25. Smith, A.G. Culture and differentiation of embryonic stem cells. Journal of tissue culture methods. 13 (2), 89-94 (1991).

26. Linkert, M. et al. Metadata matters: access to image data in the real world. The Journal of Cell Biology. 189 (5), 777-782 (2010).

27. Preibisch, S., Saalfeld, S., Tomancak, P. Globally optimal stitching of tiled 3D microscopic image acquisitions. Bioinformatics. 25 (11), 1463-1465 (2009)

28. Blin, G., Sadurska, D., Migueles, R.P., Chen, N., Watson, J.A., Lowell, S. Nessys: a novel method for accurate nuclear segmentation in 3D. bioRxiv. 502872 (2018)

29. Weiswald, L.-B., Guinebretière, J.-M., Richon, S., Bellet, D., Saubaméa, B., Dangles-Marie, V. In situ protein expression in tumour spheres: development of an immunostaining protocol for confocal microscopy. BMC cancer. 10, 106 (2010).

30. R Core Team. R: A Language and Environment for Statistical Computing. at <http://www.R-project.org/>. R Foundation for Statistical Computing. Vienna, Austria. (2013)

31. Deglincerti, A. et al. Self-organization of human embryonic stem cells on micropatterns. Nature Protocols. 11 (11), $2223-2232$ (2016).

32. Ostblom, J., Nazareth, E.J.P., Tewary, M., Zandstra, P.W. Context-explorer: Analysis of spatially organized protein expression in highthroughput screens. PLOS Computational Biology. 15 (1), e1006384 (2019).

33. Larue, L., Ohsugi, M., Hirchenhain, J., Kemler, R. E-cadherin null mutant embryos fail to form a trophectoderm epithelium. Proceedings of the National Academy of Sciences. 91 (17), 8263-8267 (1994).

34. Pieters, T., Roy, F. van Role of cell-cell adhesion complexes in embryonic stem cell biology. J Cell Sci. 127 (12), $2603-2613$ (2014).

35. Sakaue-Sawano, A. et al. Visualizing spatiotemporal dynamics of multicellular cell-cycle progression. Cell. 132 (3), $487-498$ (2008)

36. Morgani, S.M., Metzger, J.J., Nichols, J., Siggia, E.D., Hadjantonakis, A.-K. Micropattern differentiation of mouse pluripotent stem cells recapitulates embryo regionalized cell fate patterning. eLife. 7, e32839 (2018).

37. Ulman, V. et al. An objective comparison of cell-tracking algorithms. Nature Methods. 14 (12), 1141 (2017).

38. Moir, R.D., Yoon, M., Khuon, S., Goldman, R.D. Nuclear Lamins a and B1. The Journal of Cell Biology. 151 (6), 1155-1168 (2000).

39. McDole, K. et al. In Toto Imaging and Reconstruction of Post-Implantation Mouse Development at the Single-Cell Level. Cell. 175 (3), 859-876.e33 (2018).

40. Stegmaier, J. et al. Real-Time Three-Dimensional Cell Segmentation in Large-Scale Microscopy Data of Developing Embryos. Developmental Cell. 36 (2), 225-240 (2016). 